\title{
Absolute dimensions of solar-type eclipsing binaries.
}

\section{I. uvby light curves for HS Aqr, KX Aqr, AL Ari, V963 Cen, MR Del, NY Hya, DU Leo, UW LMi, and V358 Pup ${ }^{\star, \star \star}$}

\author{
J. V. Clausen, B. E. Helt, and E. H. Olsen
}

\begin{abstract}
Niels Bohr Institute for Astronomy, Physics, and Geophysics; Astronomical Observatory, Juliane Maries Vej 30, 2100 Copenhagen $\varnothing$, Denmark

Received 12 April 2001 / Accepted 22 May 2001

Abstract. We present complete uvby light curves of 9 recently discovered eclipsing binaries having late F, G, and $\mathrm{K}$ type components within or near the main-sequence band. They are the first results from a long term program carried out since 1994 at the Strömgren Automatic Telescope at ESO, La Silla. The aim is to provide the accurate absolute dimensions for solar-type stars needed for critical tests of the corresponding theoretical models. A serious dilemma appears to be present in the comparison of predictions from current stellar models with fundamental properties of known 0.7-1.1 $M_{\odot}$ eclipsing binaries (Popper 1997b, Clausen et al. 1999b). Spectroscopic observations of the 9 eclipsing binaries are either available or are being obtained, and detailed analyses of the individual systems will be published in a series of subsequent papers. The light curves were obtained as part of a systematic search for new eclipsing systems among a large sample of (mostly) spectroscopic binaries. Catalogues of the extensive uvby and $\beta$ photometry from this search will be published separately.
\end{abstract}

Key words. stars: fundamental parameters - stars: binaries: close - stars: binaries: eclipsing - stars: evolution stars: late type - techniques: photometric

\section{Introduction}

Detached, double-lined eclipsing binaries are the main source of accurate (1-2\%) data on stellar masses and radii. Few well studied systems with solar-type components are available, and accurate masses and radii for late F-K main-sequence stars are thus scarce. Andersen (1991) includes just three stars in the $0.7-1.1 M_{\odot}$ mass range (the secondary of FL Lyr and both components of HS Aur). Selection effects (small stars of low luminosity) are responsible for rather few eclipsing binaries with late-type main-sequence components being known, and for the domination of short-period systems in the sample.

In order to identify more systems, Popper (1996) undertook an impressive spectroscopic survey of known late F-K eclipsing binaries. High-quality spectroscopic orbits of 10 additional main-sequence systems have been established from this project: RT And and CG Cyg (Popper

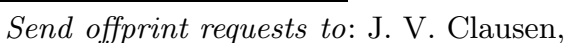

e-mail: jvc@astro.ku.dk

* Based on observations carried out with the Strömgren Automatic Telescope (SAT) at ESO, La Silla, Chile.

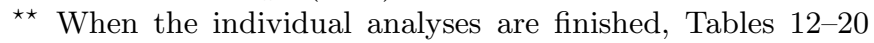
will gradually be made available in electronic form at the CDS via anonymous ftp to

cdsarc.u-strasbg.fr (130.79.128.5) or via

http://cdsweb.u-strasbg.fr/cgi-bin/qcat?J/A+A/374/980
}

1994, 1997a); UV Leo, UV Psc, and BH Vir (Popper 1997b); DU Leo (Popper 1998a); HS Aqr, V1430 Aql, HP Aur, and CV Boo (Popper 2000). Light curves exist for some of these binaries, and absolute dimensions for 9 more stars below $1.1 M_{\odot}$ have been published.

Several of the binaries mentioned above belong to the group of short-period ( $P<1$ day) RS CVn systems. They have rapidly rotating components (synchronized to the orbital period) with often fairly large relative radii $r=R / a$. Enhanced surface activity (spots) is present, leading to quite distorted light curves and consequently less welldetermined photometric elements. Attempts to fit current stellar models to the available data show systematic differences between models and binaries, which are not yet understood (Popper 1997b; Clausen et al. 1999b).

Whereas Popper (1996) searched spectroscopically for main-sequence systems among known eclipsing binaries, we have chosen to conduct a large-scale photometric search for new eclipsing cases among late-type stars known (or suspected) to be binaries. In particular, we wanted to increase the number of well-detached systems with orbital periods above 1 day. In this paper, we present uvby light curves for 9 systems; see also Clausen et al. (1999a). Analyses based on the light curves and spectroscopic observations, either new or published, will be presented in a series of forthcoming papers, and the derived absolute dimensions will be used for critical tests of theoretical 
stellar models. Catalogues of the extensive $u v b y$ and $\beta$ photometry from this search will be published separately (Olsen et al. 2001a, 2001b).

\section{Selection of candidates}

In order to be successful, a systematic photometric search for new solar-type eclipsing binaries has to be based on a comprehensive list of candidates, and large amounts of observing time at an efficient telescope at a high-quality site must be available. We have selected about 1000 (all sky) binary stars, confirmed or suspected by photometric and spectroscopic criteria, and brighter than $V \approx 9.0$, as follows:

To identify late-F to G5 main-sequence stars, we have used a critical compilation of $u v b y \beta$ photometry for about 30000 stars (Olsen 1988, 1993, 1994a, 1994b) together with unpublished $\beta$ photometry (cf. Olsen 1994b). The photometry gives rough mass and age estimates, and also $[\mathrm{Me} / \mathrm{H}]$ information. Thus, we have been able to include metal-deficient candidates with estimated masses around $1 M_{\odot}$, i.e. late-F stars significantly hotter than the Sun. Among this set of photometric candidates, we have then, from unpublished CORAVEL radial velocity information (Nordström 1994, private communication), kindly made available to us, selected both confirmed spectroscopic binaries and stars with variable radial velocity and/or high rotational velocity, indicating possible synchronization in a relatively short-period orbit.

In addition, we have included about 50 late-type spectroscopic binaries for which (preliminary) elements were available from the CfA radial velocity programs (Latham \& Torres 1995, private communication; Andersen 1996, private communication). When the Hipparcos catalog (ESA 1997) became available, we searched it for potentially interesting objects, and about 50 late-type stars, found by Hipparcos to be (possibly) eclipsing, were included. Finally, a few interesting known eclipsing binaries lacking accurate light curves, some of which are presented in this paper, were included.

\section{Observations and photometric reduction}

The photometric observations were obtained with the Strömgren Automatic Telescope (SAT) at ESO, La Silla. Details on the spectrometer and the (semi)automatic mode of the telescope are given by Olsen (1993, 1994b).

In this paper, we will only describe the differential light curve observations, which were obtained between October 1994 and March 1999, and refer to forthcoming articles for details on the uvby search observations, the $\beta$ observations, and the establishment of uvby $\beta$ standard photometry.

\subsection{Light curve observations}

Differential uvby observations were, depending on the orbital phase, done either continuously during several hours or as shorter series added to the search observations.
Table 1. rms errors of the magnitude differences (instrumental system) between the comparison stars in units of 0.1 mmag. $N$ is the total number of magnitude differences.

\begin{tabular}{lccccc}
\hline \hline Objects & $N$ & $y$ & $b$ & $v$ & $u$ \\
\hline HS Aqr & & & & & \\
HD197623, HD196218 (C1,C2) & 158 & 44 & 40 & 39 & 68 \\
HD197623, HD198585 (C1,C3) & 174 & 46 & 40 & 44 & 75 \\
HD196218, HD198585 (C2,C3) & 177 & 42 & 40 & 45 & 63 \\
\hline KX Aqr & & & & & \\
HD213845, HD213169 (C1,C2) & 743 & 39 & 39 & 37 & 53 \\
\hline AL Ari & & & & & \\
HD16707, HD17499 (C1,C2) & 231 & 52 & 58 & 54 & 83 \\
HD16707, HD15814 (C1,C3) & 200 & 53 & 58 & 60 & 73 \\
HD17499, HD15814 (C2,C3) & 225 & 49 & 46 & 45 & 64 \\
\hline V963 Cen & & & & & \\
HD115031, HD114250 (C1,C2) & 292 & 40 & 43 & 45 & 79 \\
HD115031, HD117214 (C1,C3) & 303 & 49 & 49 & 53 & 75 \\
HD114250, HD117214 (C2,C3) & 316 & 48 & 47 & 48 & 87 \\
\hline MR Del & & & & & \\
HD195633, HD195104 (C1,C2) & 183 & 44 & 41 & 42 & 64 \\
HD195633, HD197278 (C1,C3) & 185 & 47 & 46 & 47 & 68 \\
HD195104, HD197278 (C2,C3) & 175 & 38 & 40 & 43 & 69 \\
\hline NY Hya & & & & & \\
HD82074, HD80446 (C1,C2) & 564 & 47 & 46 & 49 & 75 \\
HD82074, HD80633 (C1,C3) & 516 & 47 & 49 & 54 & 69 \\
HD80446, HD80633 (C2,C3) & 552 & 47 & 47 & 49 & 76 \\
\hline DU Leo & & & & & \\
HD85087, HD83340 (C1,C2) & 166 & 58 & 62 & 67 & 84 \\
HD85087, HD83906 (C1,C3) & 184 & 57 & 63 & 64 & 86 \\
HD83340, HD83906 (C2,C3) & 165 & 63 & 68 & 71 & 86 \\
\hline UW LMi & & & & & \\
HD94218, HD94426 (C1,C2) & 232 & 64 & 68 & 71 & 87 \\
HD94218, HD91546 (C1,C3) & 224 & 75 & 80 & 83 & 99 \\
HD94426, HD91546 (C2,C3) & 138 & 67 & 75 & 78 & 95 \\
\hline V358 Pup & & & & & \\
HD53522, HD52756 (C1,C2) & 340 & 37 & 34 & 46 & 81 \\
HD53522, HD52415 (C1,C3) & 322 & 43 & 39 & 46 & 71 \\
& 333 & 36 & 34 & 44 & 70 \\
\hline
\end{tabular}

Automatic centering within a circular diaphragm of $17^{\prime \prime}$ diameter was used throughout, and all measurements were done at an airmass less than 2.1.

In general, three comparison (C) stars, preferably matching the spectral type of the binary and positioned on the sky within a few degrees from it, were adopted for each candidate. They were selected from systematic searches in the extensive uvby $\beta$ compilation referenced above (see Sect. 2), avoiding known and suspected variables as well as most visual binaries. 
Table 2. Photometric data for the eclipsing binaries and the comparison stars; see Olsen et al. (2001a, 2001b) for details. For the eclipsing binaries, the uvby information is given at maximum light level outside eclipses, at central primary eclipse, and at central secondary eclipse. The $\beta$ information is the mean values outside eclipses, see also Fig. $1 . N$ is the total number of observations used to form the mean values. $\sigma$ is the rms error (per observation) in mmag.

\begin{tabular}{|c|c|c|c|c|c|c|c|c|c|c|c|c|c|c|}
\hline Object & Phase & Sp. Type & $V$ & $\sigma$ & $b-y$ & $\sigma$ & $m_{1}$ & $\sigma$ & $c_{1}$ & $\sigma$ & $N(u v b y)$ & $\beta$ & $\sigma$ & $N(\beta)$ \\
\hline \multirow[t]{3}{*}{ HS Aqr } & 0.250 & \multirow[t]{3}{*}{ F8 } & 9.093 & 7 & 0.389 & 3 & 0.172 & 5 & 0.344 & 6 & 14 & \multirow[t]{3}{*}{2.613} & \multirow[t]{3}{*}{5} & \multirow[t]{3}{*}{4} \\
\hline & 0.000 & & 9.471 & 9 & 0.401 & 4 & 0.190 & 9 & 0.330 & 9 & 9 & & & \\
\hline & 0.500 & & 9.228 & 2 & 0.377 & 7 & 0.166 & 10 & 0.345 & 10 & 6 & & & \\
\hline HD197623 & & G5 & 7.562 & 7 & 0.412 & 5 & 0.215 & 7 & 0.405 & 8 & 253 & 2.607 & 9 & 13 \\
\hline HD196218 & & G0 & 7.420 & 7 & 0.328 & 4 & 0.159 & 6 & 0.399 & 7 & 213 & 2.630 & 2 & 3 \\
\hline HD198585 & & $\mathrm{F} 8$ & 7.673 & 6 & 0.377 & 4 & 0.128 & 8 & 0.422 & 7 & 208 & 2.602 & 5 & 3 \\
\hline \multirow[t]{3}{*}{ KX Aqr } & 0.250 & \multirow[t]{3}{*}{ F8/G0V } & 8.129 & 18 & 0.360 & 4 & 0.136 & 7 & 0.342 & 6 & 26 & \multirow[t]{3}{*}{2.592} & \multirow[t]{3}{*}{6} & \multirow[t]{3}{*}{14} \\
\hline & 0.000 & & 8.573 & 6 & 0.374 & 3 & 0.144 & 5 & 0.338 & 6 & 24 & & & \\
\hline & 0.500 & & 8.485 & 4 & 0.359 & 3 & 0.134 & 6 & 0.336 & 10 & 28 & & & \\
\hline HD213845 & & F7V & 5.215 & 7 & 0.291 & 2 & 0.161 & 4 & 0.463 & 4 & 942 & 2.669 & 5 & 20 \\
\hline HD213169 & & F6V & 7.937 & 7 & 0.307 & 4 & 0.159 & 7 & 0.452 & 7 & 742 & 2.644 & 8 & 20 \\
\hline HD213005 & & $\mathrm{F} 2 \mathrm{~V}$ & 7.357 & 16 & 0.256 & 5 & 0.155 & 6 & 0.536 & 7 & 21 & 2.683 & 6 & 17 \\
\hline \multirow[t]{3}{*}{ AL Ari } & 0.250 & \multirow[t]{3}{*}{ F8 } & 9.215 & 6 & 0.343 & 5 & 0.155 & 11 & 0.368 & 10 & 55 & \multirow[t]{3}{*}{2.625} & \multirow[t]{3}{*}{11} & 17 \\
\hline & 0.000 & & 9.766 & 12 & 0.370 & 5 & 0.168 & 7 & 0.326 & 9 & 14 & & & \\
\hline & 0.512 & & 9.449 & 4 & 0.320 & 6 & 0.151 & 10 & 0.391 & 10 & 20 & & & \\
\hline HD16707 & & F5 & 8439 & 6 & 0.332 & 5 & 0.168 & 9 & 0.383 & 9 & 324 & 2.629 & 12 & 22 \\
\hline HD17499 & & G0 & 8.198 & 6 & 0.330 & 5 & 0.158 & 9 & 0.382 & 8 & 215 & 2.620 & 7 & 20 \\
\hline HD15814 & & F8V & 6.003 & 6 & 0.355 & 3 & 0.188 & 6 & 0.378 & 5 & 209 & 2.621 & 7 & 22 \\
\hline V963 Cen & 0.550 & G2V & 8.603 & 8 & 0.409 & 6 & 0.222 & 8 & 0.321 & 7 & 26 & 2.597 & 8 & $\overline{30}$ \\
\hline & 0.000 & & 8.953 & 11 & 0.407 & 7 & 0.224 & 8 & 0.320 & 8 & 5 & & & \\
\hline & 0.289 & & 8.747 & 4 & 0.415 & 1 & 0.206 & 7 & 0.326 & 8 & 3 & & & \\
\hline HD115031 & & G2/G3V & 8.331 & 6 & 0.386 & 4 & 0.205 & 8 & 0.335 & 9 & 368 & 2.607 & 6 & 13 \\
\hline HD114250 & & F5IV/V & 7.654 & 6 & 0.323 & 4 & 0.177 & 8 & 0.483 & 10 & 238 & 2.642 & 6 & 12 \\
\hline HD117214 & & F6V & 8.073 & 6 & 0.307 & 5 & 0.173 & 9 & 0.434 & 9 & 230 & 2.645 & 7 & 13 \\
\hline MR Del & 0.250 & K0 & 8.707 & 9 & 0.520 & 3 & 0.343 & 6 & 0.219 & 11 & 11 & 2.523 & & 1 \\
\hline & 0.000 & & 9.013 & 12 & 0.527 & 7 & 0.368 & 15 & 0.212 & 11 & 9 & & & \\
\hline & 0.500 & & 8.882 & 8 & 0.516 & 4 & 0.342 & 11 & 0.220 & 15 & 7 & & & \\
\hline HD195633 & & G0Vw & 8.546 & 7 & 0.356 & 5 & 0.121 & 8 & 0.374 & 9 & 206 & 2.607 & 12 & 2 \\
\hline HD195104 & & F8 & 7.085 & 6 & 0.334 & 4 & 0.160 & 7 & 0.358 & 7 & 186 & 2.625 & 5 & 3 \\
\hline HD197278 & & G5 & 8.679 & 7 & 0.435 & 4 & 0.212 & 7 & 0.397 & 9 & 186 & 2.603 & 7 & 3 \\
\hline NY Hya & 0.250 & G5 & 8.584 & 16 & 0.444 & 4 & 0.245 & 7 & 0.350 & 7 & 42 & 2.598 & 9 & 54 \\
\hline & 0.000 & & 8.874 & 16 & 0.444 & 4 & 0.246 & 5 & 0.353 & 7 & 69 & & & \\
\hline & 0.500 & & 8.874 & 13 & 0.445 & 4 & 0.244 & 7 & 0.352 & 8 & 38 & & & \\
\hline HD82074 & & G6IV & 6.253 & 6 & 0.504 & 3 & 0.287 & 6 & 0.306 & 6 & 592 & 2.557 & 6 & 28 \\
\hline HD 80446 & & G5 & 9.144 & 7 & 0.381 & 5 & 0.199 & 8 & 0.314 & 9 & 499 & 2.596 & 6 & 24 \\
\hline HD80633 & & F8 & 7.388 & 6 & 0.307 & 4 & 0.162 & 7 & 0.424 & 7 & 472 & 2.643 & 9 & 29 \\
\hline DU Leo & 0.250 & G0 & 9.207 & 6 & 0.368 & 5 & 0.142 & 9 & 0.305 & 11 & 25 & 2.585 & 10 & 30 \\
\hline & 0.000 & & 9.989 & 26 & 0.382 & 3 & 0.153 & 9 & 0.294 & 36 & 4 & & & \\
\hline & 0.500 & & 9.884 & 17 & 0.370 & 8 & 0.129 & 9 & 0.329 & 2 & 4 & & & \\
\hline HD85087 & & F5 & 6.889 & 8 & 0.310 & 5 & 0.160 & 10 & 0.425 & 8 & 189 & 2.631 & 5 & 16 \\
\hline HD83340 & & G0IV & 7.845 & 10 & 0.398 & 6 & 0.198 & 10 & 0.450 & 10 & 143 & 2.597 & 6 & 11 \\
\hline HD83906 & & F8 & 8.214 & 7 & 0.334 & 5 & 0.175 & 9 & 0.423 & 9 & 207 & 2.624 & 7 & 13 \\
\hline UW LMi & 0.250 & G0V & 8.323 & 10 & 0.356 & 5 & 0.190 & 10 & 0.352 & 8 & 40 & 2.610 & 8 & 25 \\
\hline & 0.000 & & 8.744 & 16 & 0.361 & 5 & 0.187 & 8 & 0.344 & 9 & 5 & & & \\
\hline & 0.500 & & 8.735 & 7 & 0.370 & 6 & 0.180 & 9 & 0.350 & 10 & 7 & & & \\
\hline HD94218 & & F5 & 7.473 & 10 & 0.285 & 6 & 0.163 & 10 & 0.499 & 8 & 290 & 2.645 & 7 & 10 \\
\hline HD94426 & & F8V & 7.549 & 9 & 0.335 & 6 & 0.185 & 11 & 0.371 & 9 & 187 & 2.622 & 7 & 18 \\
\hline HD91546 & & F5V & 7.826 & 10 & 0.297 & 6 & 0.174 & 10 & 0.443 & 9 & 185 & 2.648 & 9 & 11 \\
\hline V358 Pup & 0.250 & G5V & 9.193 & 7 & 0.428 & 5 & 0.242 & 11 & 0.297 & 12 & 34 & 2.582 & 9 & 57 \\
\hline & 0.000 & & 9.561 & 5 & 0.435 & 5 & 0.246 & 12 & 0.297 & 11 & 14 & & & \\
\hline & 0.500 & & 9.391 & 5 & 0.415 & 4 & 0.226 & 8 & 0.299 & 13 & 14 & & & \\
\hline HD53522 & & K0/K1III & 8.074 & 6 & 0.476 & 4 & 0.284 & 9 & 0.324 & 9 & 543 & 2.587 & 7 & 26 \\
\hline HD52756 & & $\mathrm{K} 1 \mathrm{~V}$ & 8.448 & 5 & 0.522 & 4 & 0.462 & 8 & 0.281 & 9 & 362 & 2.563 & 8 & 26 \\
\hline HD52415 & & G3V & 8.786 & 6 & 0.398 & 5 & 0.187 & 9 & 0.325 & 9 & 355 & 2.590 & 9 & 26 \\
\hline
\end{tabular}



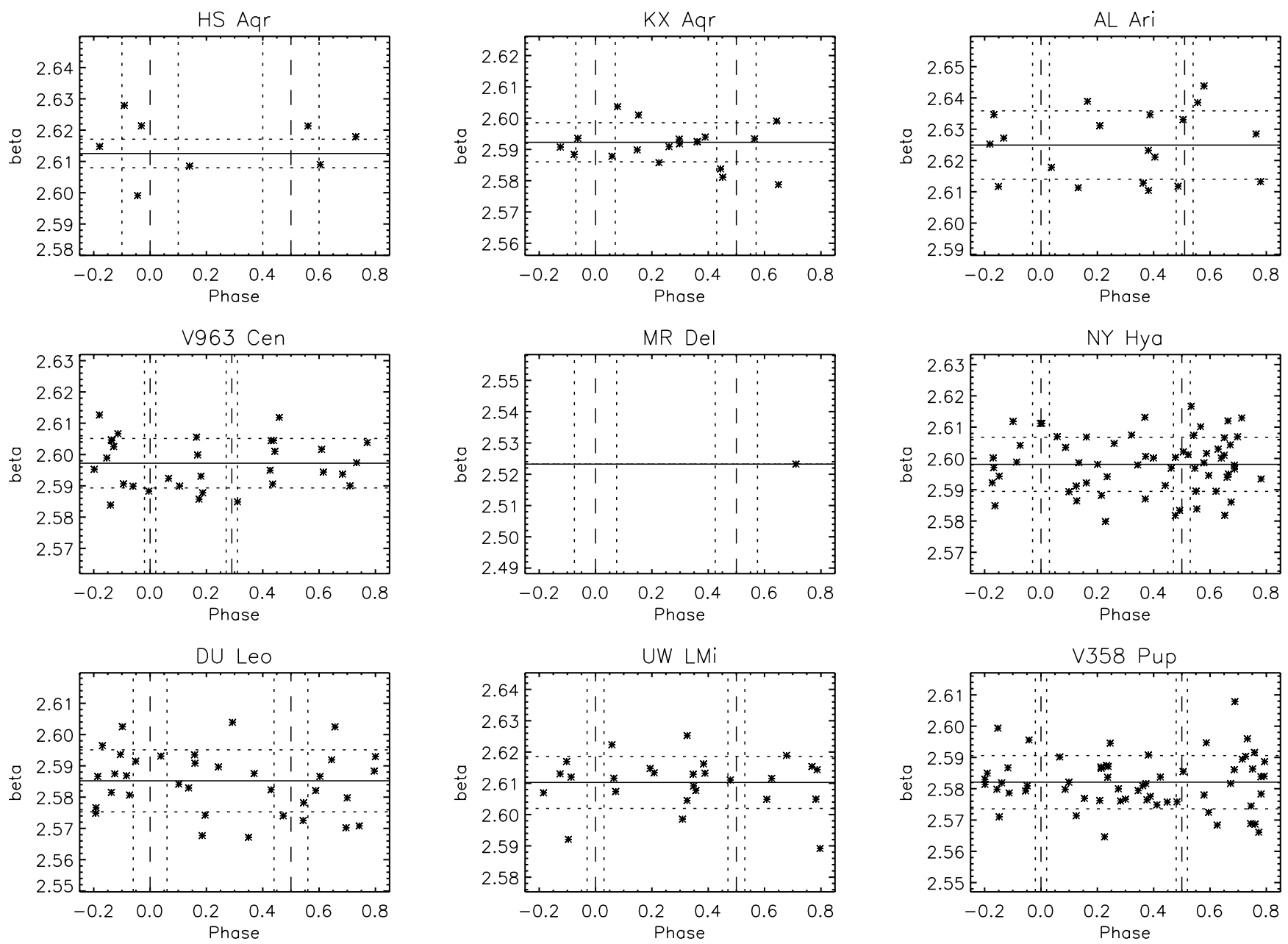

Fig. 1. Phase distribution of the $\beta$ observations, see Table 2 . The horizontal lines represent the mean value of $\beta$ outside eclipses (solid) and the rms error of one observation (dotted). The vertical lines show the location of the eclipses (dashed, central eclipses) and their duration (dotted).

The observations were done in C1-binary-C2-binaryC3-binary-C1-etc. sequences, allowing accurate magnitude differences to be formed, even if one of the comparisons was later found to be variable. An observation consisted of three individual integrations, each with an integration time of 10-90 s, and sky measurements were taken at least once per sequence, normally at a fixed position close to the binary. The number of photo-electrons counted per observation ranged between about 45000 and 500000 , depending on the binary, its phase, its airmass, the Moon, and the photometric band, leading to rms contributions from photon statistics between 5.1 and 1.5 mmag. The Heliocentric Julian Date (HJD) given for an observation refers to the midpoint of the time interval covered by the integrations.

\subsection{Photometric reduction and formation of light curves}

Linear extinction coefficients were determined individually for each night from the observations of comparison stars and other constant stars. Whenever appropriate, linear or quadratic corrections for drift during the night, caused by changes in the sky transparency and/or the influence of temperature variations on the uncooled photomultipliers, were also applied.

Since the uvby instrumental system of the SAT has proved very stable (Olsen et al. 2001a), no transformations of the light curve observations, e.g. to the standard uvby system, were needed. Differential magnitudes were formed using for each candidate observation the two comparison star observations closest in time. All comparison star observations were used with C2 and C3 first shifted to the level of C1. A careful check of the constancy of all three stars was performed. For each binary, typical rms errors per light curve point are close to those listed in Table 1 for the magnitude differences between the corresponding comparison stars.

Ephemerides were calculated from published and new times of minima, listed in Tables 3-11. In general, the new times of minima have been calculated using the method of Kwee \& van Woerden (1956).

The individual light curves are shown in Figs. 2-10 and presented in Tables 12-20, which will only be made available in electronic form. Standard uvby $\beta$ indices for 

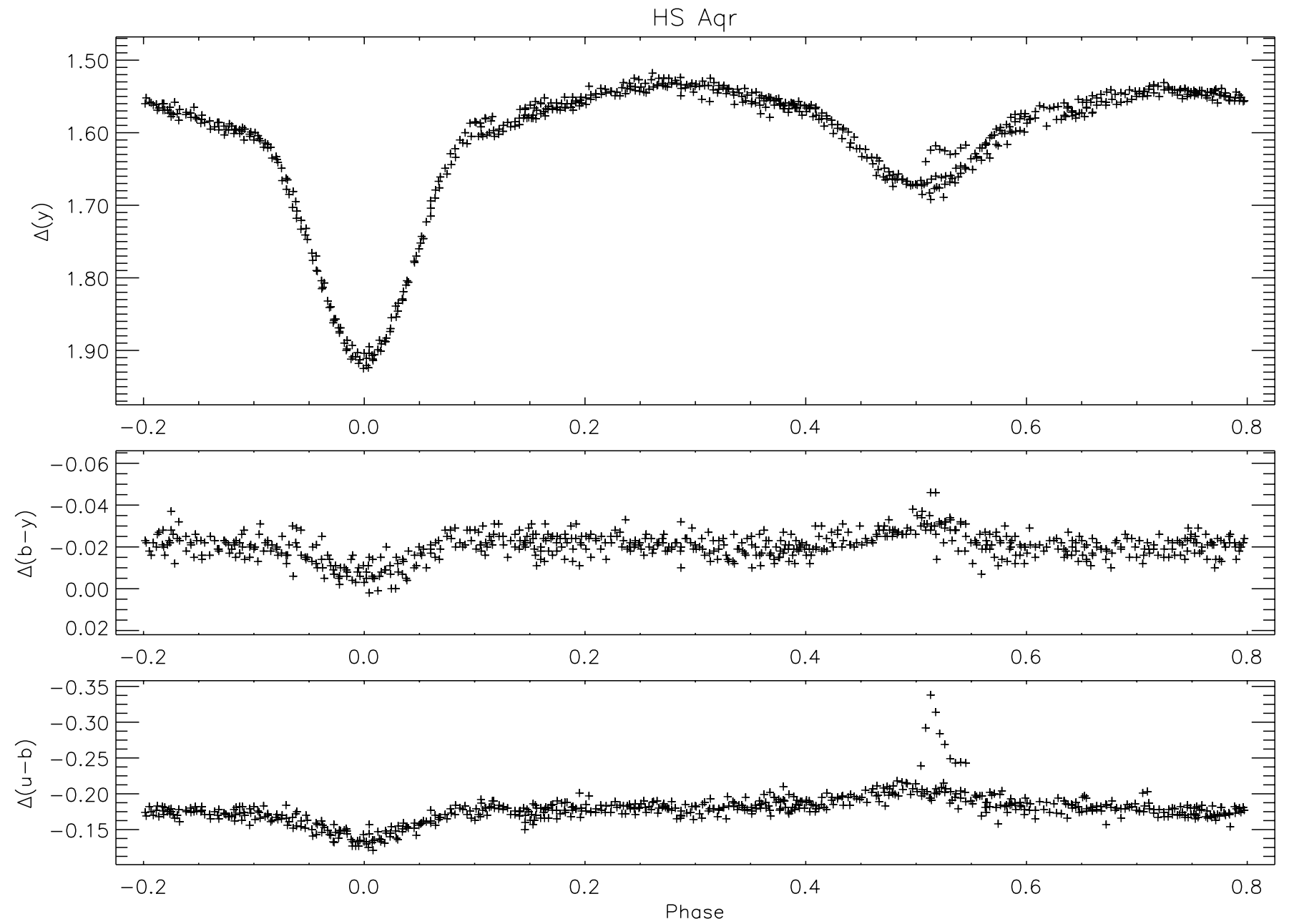

Fig. 2. $y$ light curve and $b-y$ and $u-b$ colour curves (instrumental system) for HS Aqr. Note the flare, which occurred during secondary eclipse on JD2451127.

the 9 eclipsing binaries and all comparison stars are listed in Table 2, and the phase distributions of the $\beta$ observations are shown in Fig. 1; see Olsen et al. (2001a, 2001b) for details.

\section{HS Aqr}

The X-ray source HD197010 = HS Aqr (Kazarovets et al. 1993) was discovered to be an eclipsing binary by Robb et al. (1990), who presented light curves in the $R$ and $V$ bands obtained in 1989 and 1990. Independently, Marschall et al. (1991) noticed its variability from $U B V R I$ observations and presented a $B$ light curve. Times of minima from these two studies are included in Table 3.

In his spectroscopic survey of late F-K eclipsing binaries, Popper $(1995,1996)$ mentioned HS Aqr as a potentially interesting main-sequence system, as the mass of the secondary component might well be less than $0.7 M_{\odot}$. We therefore decided to include it in the SAT observing program, although the orbital period is below 1 day and the published light curves clearly show that the components are close and quite deformed. Popper (2000) has subsequently presented accurate spectroscopic elements based on as many as 50 echelle-CCD spectra of HS Aqr. Furthermore, Nations et al. (1991) reported that 32 spectra of $1 \AA$ resolution were obtained, and additional spectroscopic observations have been done as part of the CfA radial velocity program (Stefanik 1999, private communication).

\section{1. uvby light curves}

Complete uvby light curves containing 628 points in each colour were observed on 47 nights during two periods between JD2450730 and JD2451136. HD197623, HD196218, and HD198585 were selected as comparison stars and were found to be constant within the observational accuracy of a few mmag during our observations; see Tables 1 and 2 for further information.

The light curves are shown in Fig. 2, and signs of activity are noticed from the uvby observations. Modest night-to-night variations are present, and a flare, which lasted about $20 \mathrm{~min}$, occurred during secondary eclipse on JD2451127. The levels of the two maxima at phase 0.25 and 0.75 , respectively, differ slightly in all four bands, but the differences are much smaller than observed by 
Table 3. Times of minima for HS Aqr. $O-C$ values are calculated for the ephemeris given in Eq. (1) adopting a circular orbit. M91 = Marschall et al. (1991), R90 = Robb et al. (1990).

\begin{tabular}{lrrrc}
\hline \hline HJD-2400000 & rms & Type & $O-C$ & Reference \\
\hline 48138.71960 & 0.00130 & $\mathrm{P}$ & 0.00627 & $\mathrm{R} 90$ \\
48140.85110 & 0.00040 & $\mathrm{P}$ & 0.00720 & - \\
48158.60480 & 0.00450 & $\mathrm{P}$ & 0.00617 & $\mathrm{M} 91$ \\
48182.73193 & 0.00055 & $\mathrm{P}$ & -0.01315 & - \\
48402.89996 & 0.00069 & $\mathrm{P}$ & -0.00386 & - \\
51089.55055 & 0.00010 & $\mathrm{P}$ & -0.00015 & This paper \\
51116.53850 & 0.00020 & $\mathrm{P}$ & 0.00060 & - \\
48139.79360 & 0.00150 & $\mathrm{~S}$ & 0.01499 & $\mathrm{R} 90$ \\
48144.76210 & 0.00050 & $\mathrm{~S}$ & 0.01216 & - \\
48423.86664 & 0.00189 & $\mathrm{~S}$ & 0.01223 & M91 \\
51105.53257 & 0.00080 & $\mathrm{~S}$ & 0.00261 & This paper \\
\hline
\end{tabular}

Robb et al. (1990) and Marschall et al. (1991), probably due to variations of the level of activity with time.

\subsection{Ephemeris}

Two times of primary minimum and one of secondary minimum, derived from the uvby observations, are given in Table 3 together with published times. Weighted (weight proportional to square of inverse rms) least squares fits to the times of primary and secondary minima yield periods of $0.7101895 \pm 0.0000010$ and $0.7101871 \pm 0.0000003$, respectively, and nearly identical results are obtained if equal weights are adopted. The small difference between the periods derived from primary and secondary minima, respectively, is most probably due to slight asymmetries in the eclipses arising from surface activity. Further times of minima should be obtained for a definitive determination of the ephemeris. We adopt

$$
\text { Min I }=\begin{array}{rr}
2451089.5507 \\
\pm 10
\end{array} \quad \begin{array}{r}
\text { d } 7101895 \\
\pm 10
\end{array}
$$

which represents the uvby light curves well, but does not necessarily lead to the smallest $O-C$ values, cf. Table 3. Note that the adopted period is slightly larger than those given by Robb et al. (1990) and Marschall et al. (1991). However, too few data are still available to investigate if the period should in fact be increasing with time.

\subsection{Discussion}

The uvby light curves (Fig. 2) reveal that HS Aqr is a close system consisting of two deformed components of quite different surface flux in a circular orbit. Preliminary results from the photometric analysis show that the (more massive, smaller, and hotter) primary component is well inside its Roche lobe, whereas the (less massive, larger, and cooler) secondary component is probably filling its Roche lobe. The orbital inclination is about $70^{\circ}$, and the spectroscopic elements by Popper (2000) then lead to masses of about 1.3 and $0.9 M_{\odot}$, i.e. significantly larger than the minimum masses. The secondary appears large for its mass/temperature, as already noticed by Robb et al. (1990).

In addition to the spectroscopy mentioned above, we have obtained two high-resolution spectra ( $R=48000$, 3800-8600 $\AA$ ) of HS Aqr with the FEROS spectrograph mounted at the ESO $1.52 \mathrm{~m}$ telescope (Kaufer et al. 1999), mainly for abundance studies. A detailed study of HS Aqr based on the new uvby light curves and all available spectroscopic information will be published separately.

\section{KX Aqr}

HD213379 = HIP111162 = NSV14184 = BV565 was discovered by Strohmeier et al. (1964) to be (possibly) variable and independently noted by Olsen (1983) as probably variable. The Hipparcos mission found it to be a 2 day period eclipsing binary; it subsequently was named KX Aqr (Kazarovets et al. 1999). The Hipparcos parallax measurement leads to a distance of about 160 pc. CORAVEL observations (Nordström 1994, private communication) showed it to be double-lined, and based on this information, uvby indices (Olsen 1983), and a spectral type of F8/G0V, it was included in our search for FGK main-sequence eclipsing binaries from the first night and, independently of the Hipparcos observations, discovered to be eclipsing in 1994 .

\section{1. uvby light curves}

Complete uvby light curves containing as many as 1662 points in each colour were observed on 90 nights during three seasons between JD2447881 and JD2451170. HD213845, HD213169, and HD213005 were selected as comparison stars. The two first stars were found to be constant within the observational accuracy of a few mmag during our observations; see Tables 1 and 2 for further information. However, after a couple of nights, signs of variability at the level of $\pm 10-15 \mathrm{mmag}$ were seen for HD213005, and it was consequently rejected as comparison.

The light curves are shown in Fig. 3, and surface activity is clearly seen from the uvby observations. We have therefore decided to observe three independent sets of uvby light curves, separated in time by about one year. As mentioned in Sect. 5.3, lines from a third bright component are present in the spectra of KX Aqr, and larger fractions of the components than indicated by the modest observed depths are therefore obscured during the eclipses.

\subsection{Ephemeris}

From the uvby observations presented here, supplemented by observations on JD2451878, five times of primary and six times of secondary minimum are available, see Table 4. The two times of secondary minimum at JD2450763 and 

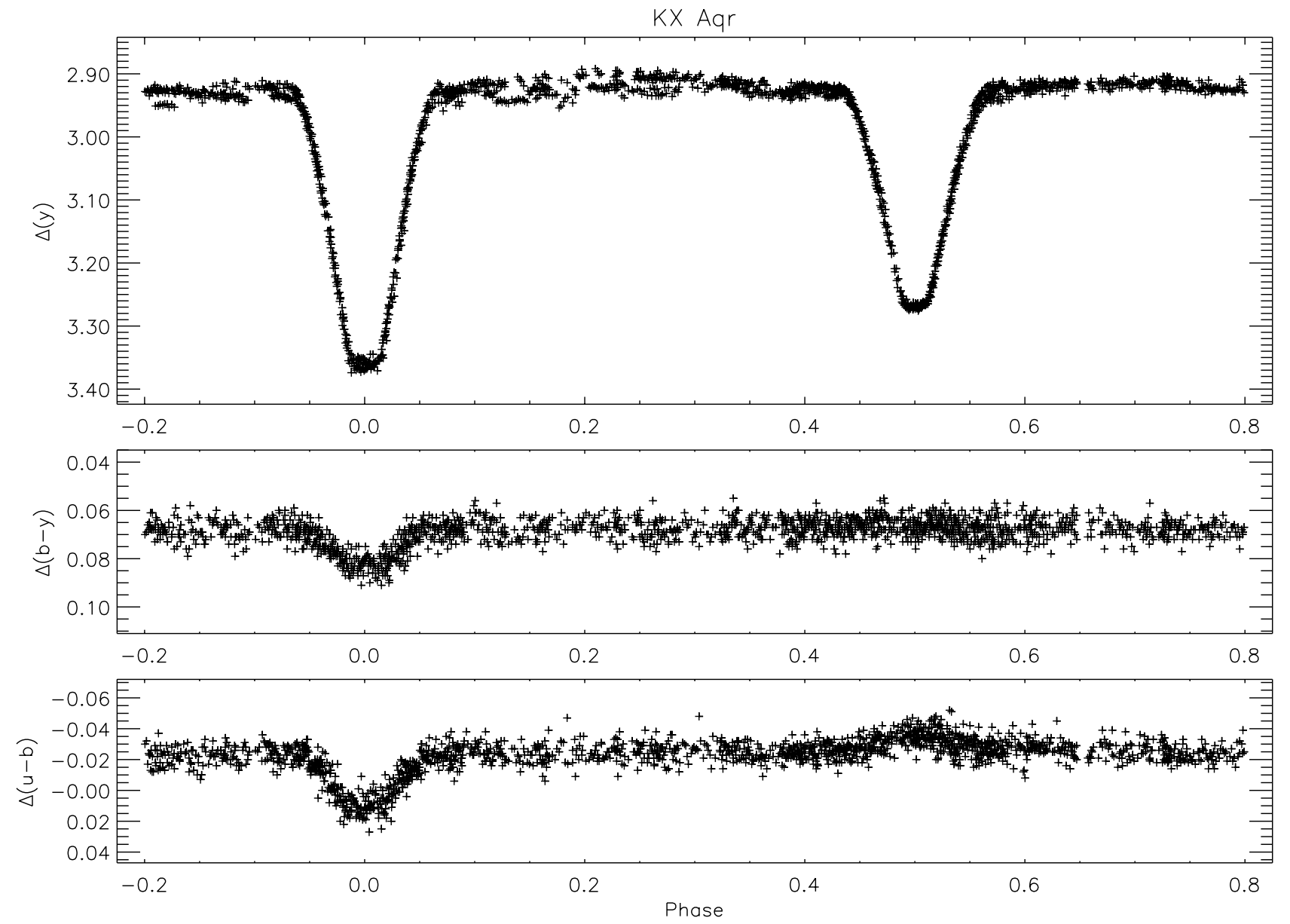

Fig. 3. $y$ light curve and $b-y$ and $u-b$ colour curves (instrumental system) for KX Aqr.

Table 4. Times of minima for KX Aqr determined from the uvby observations. $O-C$ values are calculated for the ephemeris given in Eq. (2) adopting a circular orbit. Times of three near mid-eclipse observations from the Hipparcos photometry (ESA 1997) are included but not used for the ephemeris calculation.

\begin{tabular}{lrcrc}
\hline \hline HJD-2400000 & rms & Type & $O-C$ & Reference \\
\hline 47881.28159 & & $\mathrm{P}$ & 0.00909 & ESA 1997 \\
48053.45523 & & $\mathrm{P}$ & 0.01232 & - \\
50428.57200 & 0.00200 & $\mathrm{P}$ & -0.00395 & This paper \\
51100.68000 & 0.00200 & $\mathrm{P}$ & 0.01400 & - \\
51102.73600 & 0.00200 & $\mathrm{P}$ & -0.00435 & - \\
51154.59200 & 0.00400 & $\mathrm{P}$ & -0.00715 & - \\
51878.54300 & 0.00500 & $\mathrm{P}$ & -0.00500 & - \\
48763.92645 & & $\mathrm{~S}$ & 0.01798 & ESA 1997 \\
50427.54500 & 0.00200 & $\mathrm{~S}$ & 0.00622 & This paper \\
50429.60300 & 0.00400 & $\mathrm{~S}$ & -0.01013 & - \\
50763.58420 & 0.00050 & $\mathrm{~S}$ & 0.00040 & - \\
51124.53000 & 0.00400 & $\mathrm{~S}$ & 0.00895 & - \\
51126.60000 & 0.00100 & $\mathrm{~S}$ & 0.00460 & - \\
51153.56100 & 0.00200 & $\mathrm{~S}$ & -0.00098 & - \\
\hline
\end{tabular}

JD2451126 are of sufficient accuracy, whereas the other determinations are based on few observations near the central eclipses and consequently quite large errors have been assigned. Comparing weighted and unweighted linear least squares fits to both types of minima we adopt the following ephemeris:

$$
\begin{array}{rrr}
\text { Min } I=\quad 2451100.6660 & + & 2 \mathrm{~d} .074352 \\
\pm 30 & \pm E
\end{array}
$$

Although based on several times of minima of rather low quality this ephemeris represents the uvby light curve observations from the three observing periods very well. Secondary eclipse occurs at phase 0.5 suggesting a circular orbit.

A significantly longer period of 2 d 07441 was derived from the Hipparcos photometry. It should, however, be pointed out that possible light-time effects due to the third component have not been considered. Observation of further times of minima should be done.

\subsection{Discussion}

The new uvby light curves show that KX Aqr consists of two well-detached components of different surface flux in a presumably circular orbit. One or both components exhibit surface activity. October 1998 - August 1999 (JD2451102 - 2451392), about 25 high-resolution spectra $(R=48000,3800-8600 \AA)$ of KX Aqr were 

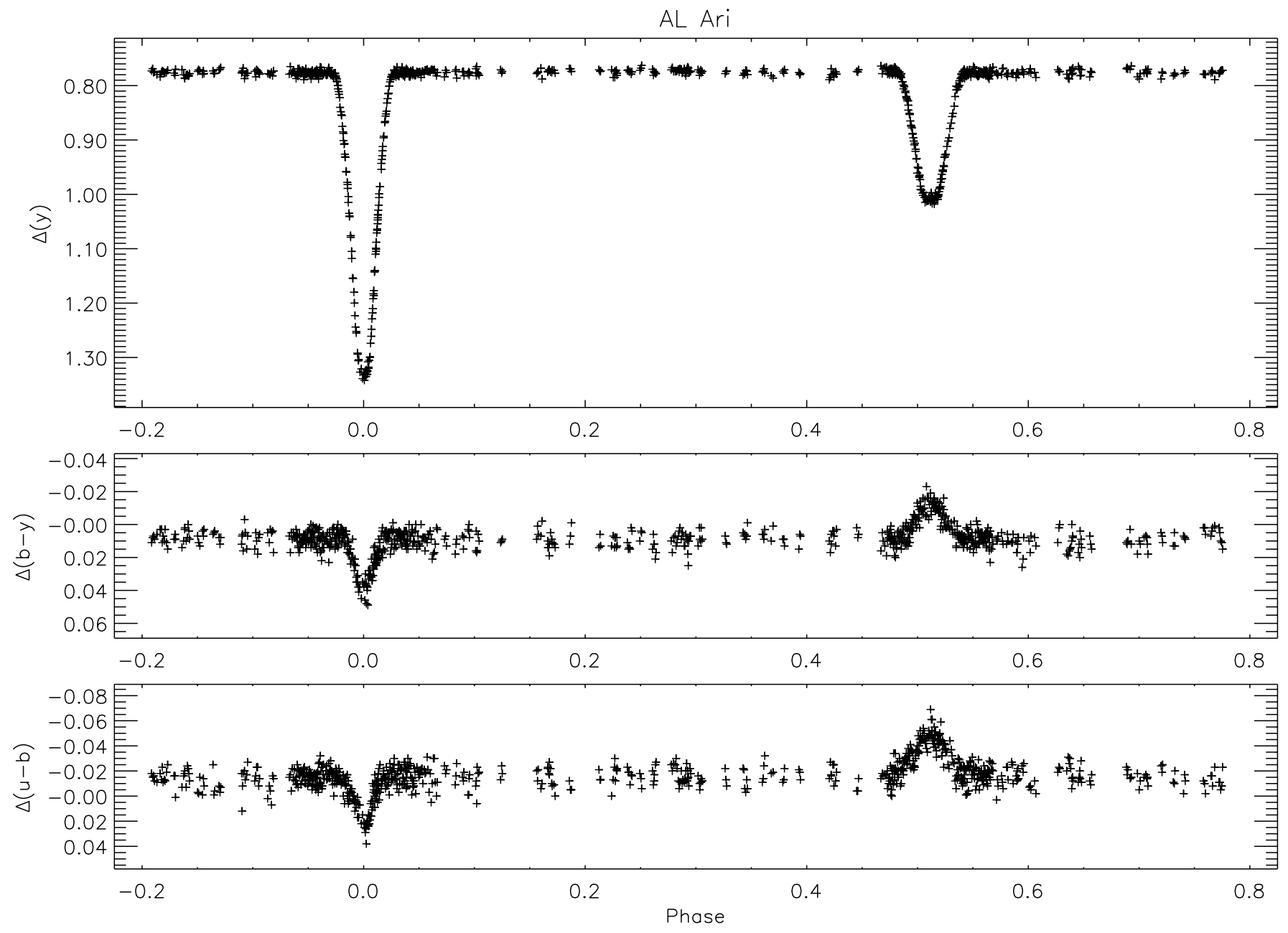

Fig. 4. $y$ light curve and $b-y$ and $u-b$ colour curves (instrumental system) for AL Ari.

obtained with the FEROS spectrograph mounted at the ESO 1.52m telescope (Kaufer et al. 1999). We expect to obtain both accurate spectroscopic elements and abundances from these observations. In addition, about 30 radial velocity measurements have been obtained from the CORAVEL project (Udry 2000, private communication).

However, the analysis is complicated by a third set of quite strong, sharp lines in addition to the two sets of slightly broadened lines from the eclipsing components. The Hipparcos catalog (ESA 1997) lists only one component for KX Aqr, which does not have a known visual companion, and additional radial velocity observations, obtained at a different epoch, are needed in order to clarify if $\mathrm{KX}$ Aqr is in fact member of a triple system.

\section{AL Ari}

$\mathrm{BD}+12^{\circ} 378=\mathrm{SAO} 93068=\mathrm{HIP} 12657$ was discovered by the Hipparcos mission to be an eclipsing binary (ESA 1997; Perryman 1999) and subsequently got the variable name AL Ari (Kazarovets et al. 1999). 47 Hipparcos photometric observations are available and 4 of them, all obtained at JD2448478, are significantly fainter than the rest. The ephemeris could of course not be established from these data. We decided to include it in the SAT program during 1997, mainly because of its F8 spectral type, but also because of its modest distance of about 100 pc (ESA 1997). Already less than two weeks after the first uvby observation of AL Ari had been obtained, the first sign of an eclipse was observed (on JD2450741).

\section{1. uvby light curves}

HD16707, HD17499, and HD15814 were selected as comparison stars and were all found to be constant within the observational accuracy of a few mmag during our observations; see Tables 1 and 2 for further information. The C3 comparison star, the bright nearby F8V object HD15814 = HR741 = 29 Ari, is a known double-lined spectroscopic binary (Lu 1986), but as expected from the long orbital period of 19.4 and low $M \sin ^{3} i$ values of 0.149 and $0.115 M_{\odot}$, no signs of eclipses or sinusoidal variations are present.

Complete uvby light curves (793 points per band) were obtained on 84 nights between JD2450741 and JD2451161. As seen in Fig. 4, AL Ari consists of two well-detached and quite different components in a slightly eccentric orbit. 
Table 5. Times of minima for AL Ari. $O-C$ values $(\mathrm{P})$ and phases (S) are calculated for the ephemeris given in Eq. (3).

\begin{tabular}{lrcrc}
\hline \hline HJD-2400000 & rms & Type & $O-C / \mathrm{Ph}$ & Reference \\
\hline 51112.80746 & 0.00010 & $\mathrm{P}$ & 0.00000 & This paper \\
51129.71510 & 0.00020 & $\mathrm{~S}$ & 0.51176 & - \\
51144.70350 & 0.00100 & $\mathrm{~S}$ & 0.51137 & - \\
\hline
\end{tabular}

The light curves show no indication of surface activity of the components.

\subsection{Ephemeris}

From the uvby observations, one time of primary and two times of secondary minimum are available, see Table 5, whereas no previous determinations have been published. The orbital period was determined by the method of Lafler \& Kinman (1965), and we adopt the ephemeris

$\operatorname{Min} \mathrm{I}=2451112.80746+3^{\mathrm{d}} .747462 \times E$.

Secondary eclipse occurs at phase 0.512 , and a small orbital eccentricity of $e \approx 0.06$ is found. Based on preliminary absolute dimensions, the apsidal motion period is judged to be long, of the order of a few thousand years, but the relativistic contribution to the motion is expected to be significant. Anyhow - additional times of minima for AL Ari should be obtained during the coming decades in order to establish more firmly its ephemeris.

\subsection{Discussion}

The new uvby light curves and uvby standard indices show that AL Ari, consisting of two well-detached and quite different components, is among the very few known systems suitable for reliable tests of theoretical stellar models around and below $1 M_{\odot}$ (Clausen et al. 1999b). Therefore, in order to obtain accurate absolute dimensions and abundances, AL Ari has been included in the spectroscopic part of our project. About 30 high-resolution spectra $(R=48000,3800-8600 \AA)$ have been obtained with the FEROS spectrograph mounted at the ESO $1.52 \mathrm{~m}$ telescope (Kaufer et al. 1999) and are presently being analysed. A detailed study of AL Ari based on the new uvby light curves and the new spectroscopic observations will be published separately.

\section{V963 Cen}

The G2V object HD115496 was observed by Olsen (1993) and noted as a probable variable based on two very discordant observations. It was subsequently included in the CORAVEL radial velocity program, where it was discovered to be a double-lined spectroscopic binary consisting of two $\approx 1 M_{\odot}$ components in a 16-day period and highly eccentric $(e \approx 0.42$ ) orbit (Andersen 1995, private communication). It was thereby included in the present project and soon discovered to be an eclipsing binary.
Table 6. Times of minima for V963 Cen. $O-C$ values $(\mathrm{P})$ and phases $(\mathrm{S})$ are calculated for the ephemeris given in Eq. (4).

\begin{tabular}{lrrrc}
\hline \hline HJD-2400000 & rms & Type & $O-C / \mathrm{Ph}$ & Reference \\
\hline 50820.80104 & 0.00030 & $\mathrm{P}$ & 0.00000 & This paper \\
51217.80515 & 0.00010 & $\mathrm{P}$ & 0.00000 & - \\
50855.76512 & 0.00060 & $\mathrm{~S}$ & 0.28982 & - \\
51252.77112 & 0.00030 & $\mathrm{~S}$ & 0.28994 & - \\
\hline
\end{tabular}

The Hipparcos mission independently detected light variations, and based on this discovery HD115496 got the variable name V963 Cen (Kazarovets et al. 1999). The ephemeris could, however, not be derived from the Hipparcos data; see Sect. 7.2. A distance of about 90 pc is obtained from the Hipparcos parallax determination.

\section{1. uvby light curves}

HD115031, HD114250, and HD117214 were selected as comparison stars and found to be constant within the observational accuracy of a few mmag during our observations; see Tables 1 and 2 for further information. Complete uvby light curves (975 points per band) were obtained on 110 nights during three observing periods between JD2450502 and JD2451253. As seen in Fig. 5, V963 Cen consists of two extremely well-detached components in an eccentric orbit. Indications of slight variability, probably due to spots, are noticed and will be studied in detail in the forthcoming analysis.

\subsection{Ephemeris}

From the uvby observations, two times of primary and two times of secondary minimum are available, see Table 6, whereas no previous determinations have been published. We adopt the following ephemeris determined from the times of primary minimum:

Min I $=2451217.80515+15.269389 \times E$.

From the Hipparcos mission more than 200 photometric observations exist, and observations during the eclipses were obtained at JD2448163 (primary), JD2448763 (secondary), and JD2448957 (primary), and the observation at JD2448163.64343 is judged to be very close to central primary eclipse. Furthermore, preliminary spectroscopic elements from the CORAVEL radial velocities (Andersen 1995, private communication) yield an epoch of $2448392.990 \pm 0.012$ and a period of 15 d $26936 \pm 0.0004$. Within their errors, these results all support the ephemeris given above. Applying the method of Lafler \& Kinman (1965) to the $u v b y$ light curves, a slightly longer period of 15 . 26942 is obtained, but the result may well be influenced by the small light variations noticed for V963 Cen. 

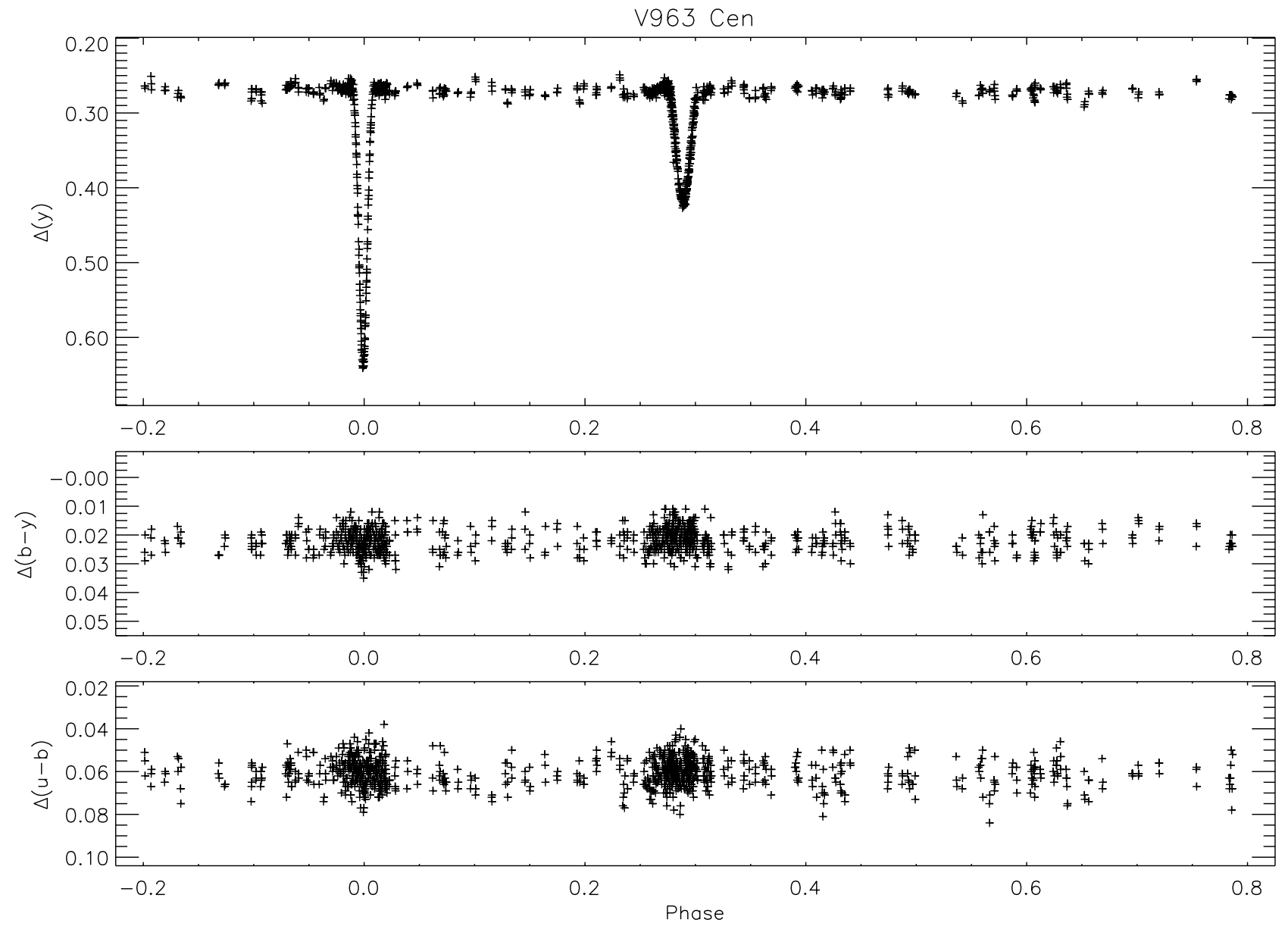

Fig. 5. $y$ light curve and $b-y$ and $u-b$ colour curves (instrumental system) for V963 Cen.

\subsection{Discussion}

Preliminary results from the analyses of the uvby light curves and the complete sample of CORAVEL radial velocities (about 45 per component, Udry 1999, private communication) show that V963 Cen consists of two nearly identical components. The quite large difference in the depths of primary and secondary eclipse (see Fig. 5) is therefore primarily caused by the high eccentricity of the orbit $(e \approx 0.42)$ and its orientation. Due to the very small relative radii of the components (about 0.04), the apsidal motion period is very long, probably more than $50000 \mathrm{yr}$, and the relativistic contribution is judged to be about twice the classical one.

For abundance studies, we have obtained a few highdispersion spectra $(R=48000,3800-8600 \AA)$ with the FEROS spectrograph mounted at the ESO $1.52 \mathrm{~m}$ telescope (Kaufer et al. 1999). A detailed study of V963 Cen based on the new uvby light curves, the CORAVEL radial velocities, and these spectra will be published separately.

\section{MR Del}

HD195434 = ADS13940 = HIP101236 is a high proper motion visual binary ( $\rho=1$ '"8, Germain et al. 1999) and a soft X-ray source (Pye et al. 1995). The brighter com- ponent was discovered by Cutispoto et al. (1997) to be a short-period eclipsing binary, and they obtained $U B V R I$ light curves (the visual companion included) showing clearly BY Dra-type variability. The eclipsing system was later named MR Del (Kazarovets et al. 1999). 67 photometric observations were obtained by the Hipparcos project, and a distance of just about $44 \mathrm{pc}$ was determined (ESA 1997), making MR Del a potentially interesting system for the definition of the radiative flux relation (Popper 1998b). Hipparcos gives a photometric period of $0 \mathrm{~d} 521690$ and a magnitude difference between the visual components of 0.28 .

We decided to include the binary in the uvby program, since it is expected to be old and metal-deficient, and since low masses of about $0.7 M_{\odot}$ were estimated by Cutispoto et al. (1997).

\section{1. uvby light curves}

Complete uvby light curves (601 points per band) were obtained on 35 nights during three observing periods between JD2450727 and JD2451114. HD195633, HD195104, and HD197278 were selected as comparison stars and found to be constant within the observational accuracy of a few mmag during our observations; see Tables 1 

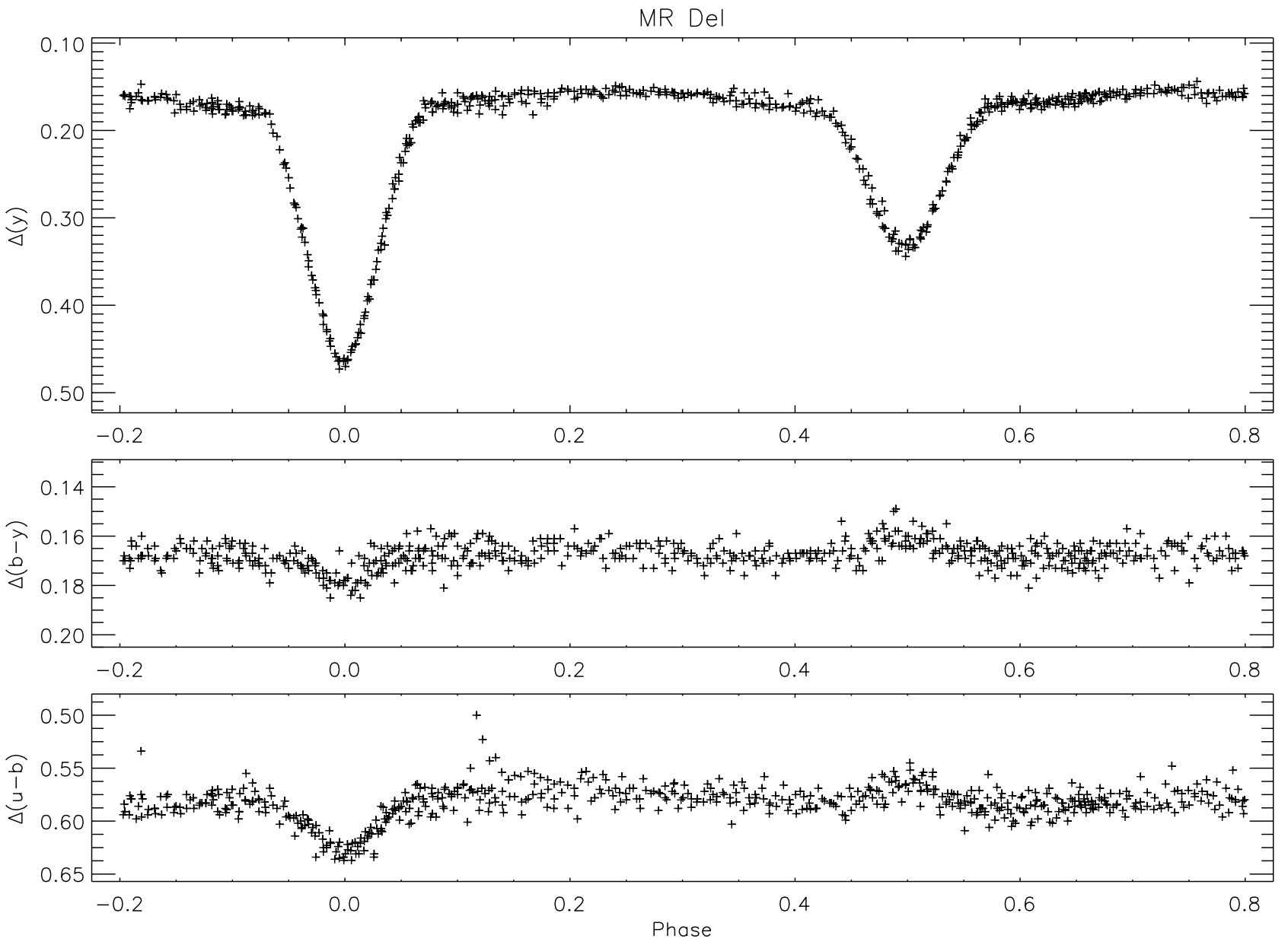

Fig. 6. $y$ light curve and $b-y$ and $u-b$ colour curves (instrumental system) for MR Del. A flare, which occurred on JD2451097 near phase 0.12 , is seen in the $u-b$ panel.

and 2 for further information. One of the comparison stars, HD195633, is a well known metal-deficient star (e.g. Cayrel de Strobel et al. 1997) with uvby photometry obtained by Schuster \& Nissen (1988) and Olsen (1993, 1994b). The new results presented in Table 2 agree closely with the previous data.

As seen in Fig. 6, MR Del consists of two components of somewhat different surface flux in a circular, shortperiod orbit. A significant amount of third light from the close visual companion is present, causing fairly shallow eclipses. Night-to-night variability due to surface activity is clearly seen from the photometry, increasing in strength from $y$ to $u$, but not at a level comparable to that observed by Cutispoto et al. (1997). Also, the asymmetries with respect to the central eclipses are less pronounced. As for HS Aqr (see Fig. 2), a flare was also observed for MR Del. It occurred on JD2451097, lasted for about $25 \mathrm{~min}$ and is most clearly seen in the $u$ band (Fig. 6, $u-b$ panel near phase 0.12).

\subsection{Ephemeris}

The uvby observations allow six times of primary and five times of secondary minimum to be derived, see Table 7.
From a weighted least squares fit to the times of primary minimum plus the epochs given by Cutispoto et al. (1997) and in the Hipparcos catalog (ESA 1997), we derive the following ephemeris:

$$
\text { Min I = 2451098.52806 }+\quad 0.52169000 \quad \times E .
$$

The period agrees well with the Hipparcos result and with that determined by Cutispoto et al. (1997), 0.52175 \pm 0 d00022, but is much more accurate. Applying only the primary minima from the uvby observations, a nearly identical but less accurate period of $0.5216889 \pm 0.0000060$ is obtained.

\subsection{Discussion}

MR Del is interesting as an example of a halo population or old disk short period binary with probably variable and sometimes significant surface activity and variable X-ray flux. Physical parameters for the components have been determined by Cutispoto et al. (1997) from their October 1995 light curves, but these results need to be refined by independent photometric and spectroscopic analyses. 
Table 7. Times of minima for MR Del. $O-C$ values are calculated for the ephemeris given in Eq. (5) adopting a circular orbit. The epochs listed by Cutispoto et al. 1997 (C97) and the Hipparcos catalog (ESA 1997) have been included, assuming an error of 0 d. 001 .

\begin{tabular}{lrrrc}
\hline \hline HJD-2400000 & rms & Type & $O-C$ & Reference \\
\hline 48500.51600 & 0.00100 & $\mathrm{P}$ & 0.00414 & ESA 1997 \\
49950.27900 & 0.00100 & $\mathrm{P}$ & -0.00937 & $\mathrm{C} 97$ \\
50925.84900 & 0.00100 & $\mathrm{P}$ & 0.00033 & This paper \\
50926.89260 & 0.00200 & $\mathrm{P}$ & 0.00055 & - \\
51098.52815 & 0.00020 & $\mathrm{P}$ & 0.00009 & - \\
51100.61483 & 0.00020 & $\mathrm{P}$ & 0.00001 & - \\
51110.52729 & 0.00020 & $\mathrm{P}$ & 0.00036 & - \\
51111.57004 & 0.00020 & $\mathrm{P}$ & -0.00027 & - \\
50920.89420 & 0.00400 & $\mathrm{~S}$ & 0.00159 & - \\
50931.84869 & 0.00030 & $\mathrm{~S}$ & 0.00058 & - \\
51092.52886 & 0.00030 & $\mathrm{~S}$ & 0.00024 & - \\
51094.61536 & 0.00030 & $\mathrm{~S}$ & -0.00002 & - \\
51104.52727 & 0.00020 & $\mathrm{~S}$ & -0.00022 & - \\
\hline
\end{tabular}

Parallel to the observation of uvby light curves, spectroscopic observations have been obtained at the MMT (Torres 1998, private communication), and a study of MR Del based on both data sets will be published separately.

\section{NY Hya}

HD80747 = HIP45887 was discovered to be an eclipsing binary by the Hipparcos mission (ESA 1997) and has been named NY Hya (Kazarovets et al. 1999). A distance of about 100 pc was obtained from the Hipparcos astrometry. Based on the Hipparcos discovery (wrong orbital period, see Sect. 9.2), uvby indices (Olsen 1993), and the G5 spectral type, we decided to add the system to the uvby program. Ribas (1999), knowing from the Hipparcos data that NY Hya is an eclipsing binary, obtained about 15 spectroscopic observations with the CORAVEL scanner at Observatoire de Haute Provence and discovered that NY Hya is a double-lined system consisting of two solartype stars of nearly identical masses. In total, about 25 radial velocities for each component are available from the CORAVEL project (Udry 1999, private communication).

\section{1. uvby light curves}

Complete light curves (1799 points per band) of NY Hya were obtained during two observing periods from JD2450775 to JD2451253, and as seen in Fig. 7 it consists of two well-detached components of comparable surface fluxes in a circular orbit. Variability due to surface activity (spots) is clearly seen, and in order to obtain photometric elements free from systematic errors introduced by the spots, and also to try to determine their patterns with time, we decided to obtain complete light curves during each of the two observing periods.
Table 8. Times of minima for NY Hya. $O-C$ values are calculated for the ephemeris given in Eq. (6), adopting a circular orbit.

\begin{tabular}{lrrrc}
\hline \hline HJD-2400000 & rms & Type & $O-C$ & Reference \\
\hline 50845.68650 & 0.00020 & $\mathrm{P}$ & 0.00024 & This paper \\
51160.77380 & 0.00015 & $\mathrm{P}$ & -0.00141 & - \\
51203.74250 & 0.00010 & $\mathrm{P}$ & 0.00062 & - \\
51227.60970 & 0.00100 & $\mathrm{P}$ & -0.00256 & - \\
51246.70840 & 0.00020 & $\mathrm{P}$ & -0.00016 & - \\
50833.75200 & 0.00020 & $\mathrm{~S}$ & 0.00093 & - \\
50876.71950 & 0.00030 & $\mathrm{~S}$ & 0.00176 & - \\
51234.77210 & 0.00100 & $\mathrm{~S}$ & -0.00127 & - \\
\hline
\end{tabular}

HD82074, HD80446, and HD80633 were selected as comparison stars and found to be constant within the observational accuracy of a few mmag; see Tables 1 and 2 for further information. HD82074 = HR3762 was also used as comparison star by Lockwood et al. (1997) in their longterm study of photometric variability of Sun-like stars and was found to be constant in this study.

\subsection{Ephemeris}

From the uvby observations, five times of primary and three times of secondary minimum are available, see Table 8, and a weighted least squares fit to the times of primary minimum yields the following ephemeris:

$$
\text { Min I }=\begin{array}{rrr}
2451203.74188 & +\quad 4.774075 & \times E . \\
\pm 52 & \pm 19 &
\end{array}
$$

The period agrees with that obtained by Ribas (1999) (4.774) and the ephemeris represents perfectly the Hipparcos data. Thus, the orbital period is three times larger than originally announced from the 66 Hipparcos observations.

\subsection{Discussion}

As mentioned in Sect. 1, few well-detached eclipsing binaries with solar-type components are available for critical tests of the corresponding stellar models. NY Hya is therefore a priori an important system, but since surface activity is present, and since the components are nearly identical, very detailed and accurate observations are needed in order to derive reliable absolute dimensions. We have therefore, as a supplement to the CORAVEL data, obtained about 40 high-resolution spectra $(R=48000$, 3800-8600 $\AA$ ) of NY Hya with the FEROS spectrograph mounted at the ESO $1.52 \mathrm{~m}$ telescope (Kaufer et al. 1999). Besides providing independent spectroscopic orbits, abundances and luminosity ratios between the components will be determined from these observations. A detailed study of NY Hya will be published separately (Clausen et al. 2001). 

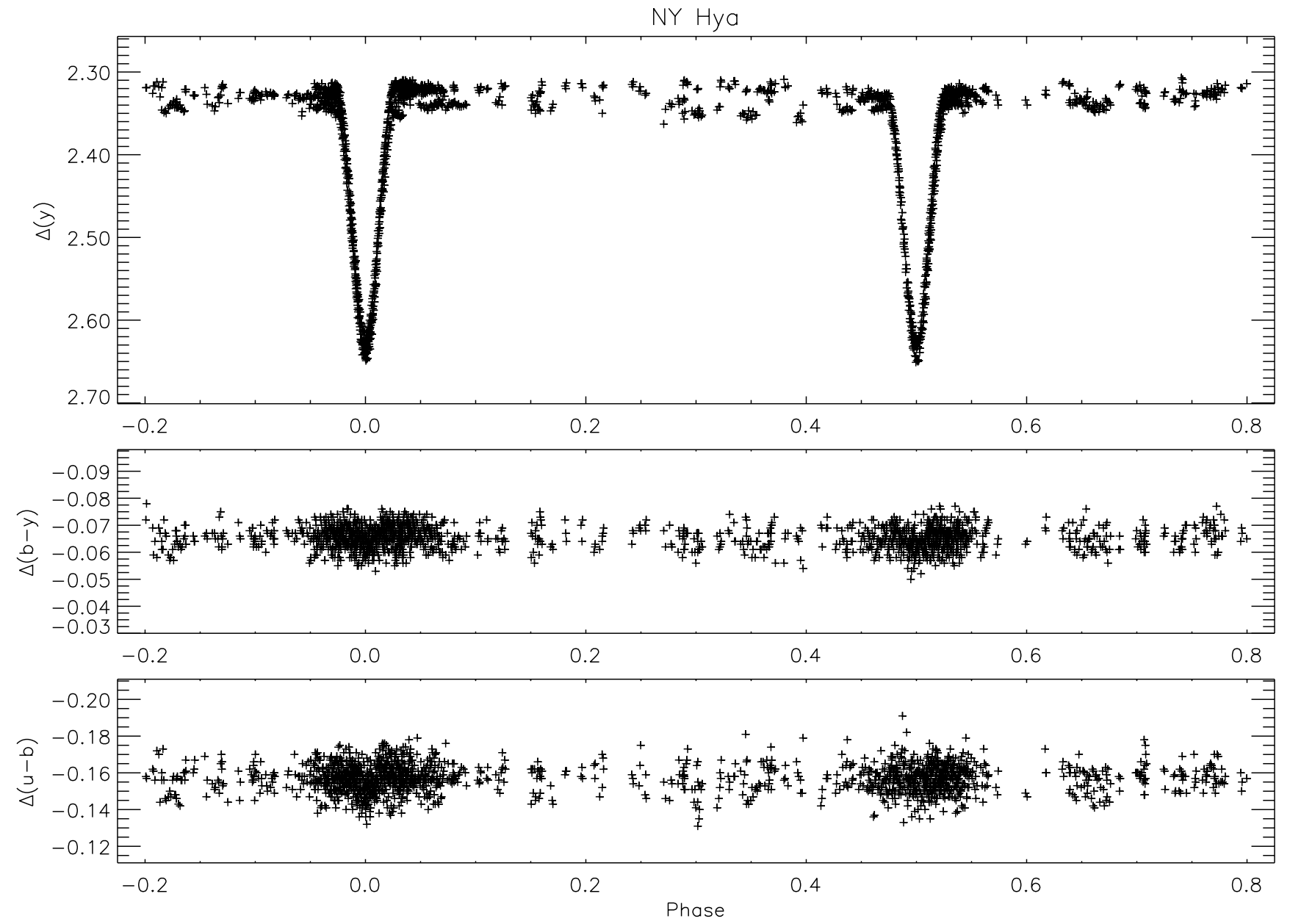

Fig. 7. $y$ light curve and $b-y$ and $u-b$ colour curves (instrumental system) for NY Hya.

\section{DU Leo}

HD84207 = DU Leo (Kazarovets et al. 1993) was discovered to be an eclipsing binary by Kaiser (1990), and Williams et al. (1990) presented $R$-band differential photometry. Preliminary masses were obtained by Popper (1993), who later published an accurate spectroscopic orbit and average absolute dimensions (Popper 1998a). From the available light curves, only approximate average photometric elements for the components could be determined. Average dimensions of $0.93 M_{\odot}$ and $1.18 R_{\odot}$ were obtained, and DU Leo was found to be quite old.

\section{1. uvby light curves}

Complete $u v b y$ light curves containing 618 points in each colour were obtained on 53 nights during two observing periods between JD2450780 and JD2451253. HD85087, HD83340, and HD83906 were selected as comparison stars and were found to be constant within the observational accuracy of a few mmag during our observations; see Tables 1 and 2 for further information.

The light curves, which are shown in Fig. 8, clearly reveal a detached system (relative radii about 0.19 ) consisting of two slightly different components in a circular
Table 9. Times of minima for DU Leo. $O-C$ values are calculated for the ephemeris given in Eq. (7) adopting a circular orbit. A list of previous times of minima, 40 photographic and 2 photoelectric, is given by Williams (1994).

\begin{tabular}{lrrrc}
\hline \hline HJD-2400000 & rms & Type & $O-C$ & Reference \\
\hline 50798.82908 & 0.00020 & $\mathrm{P}$ & -0.00069 & This paper \\
50842.80372 & 0.00030 & $\mathrm{P}$ & 0.00003 & - \\
50860.66812 & 0.00010 & $\mathrm{P}$ & 0.00002 & - \\
50871.66170 & 0.00010 & $\mathrm{P}$ & 0.00012 & - \\
50904.64149 & 0.00030 & $\mathrm{P}$ & -0.00053 & - \\
51231.69818 & 0.00010 & $\mathrm{P}$ & 0.00009 & - \\
50873.72283 & 0.00015 & $\mathrm{~S}$ & -0.00003 & - \\
50880.59425 & 0.00030 & $\mathrm{~S}$ & 0.00047 & - \\
50902.58103 & 0.00010 & $\mathrm{~S}$ & 0.00028 & - \\
\hline
\end{tabular}

orbit. Surface activity is noticed from the light curve observations, and the general light levels during the two observing periods differ.

\subsection{Ephemeris}

Six times of primary minimum and three of secondary minimum derived from the uvby observations are given in 

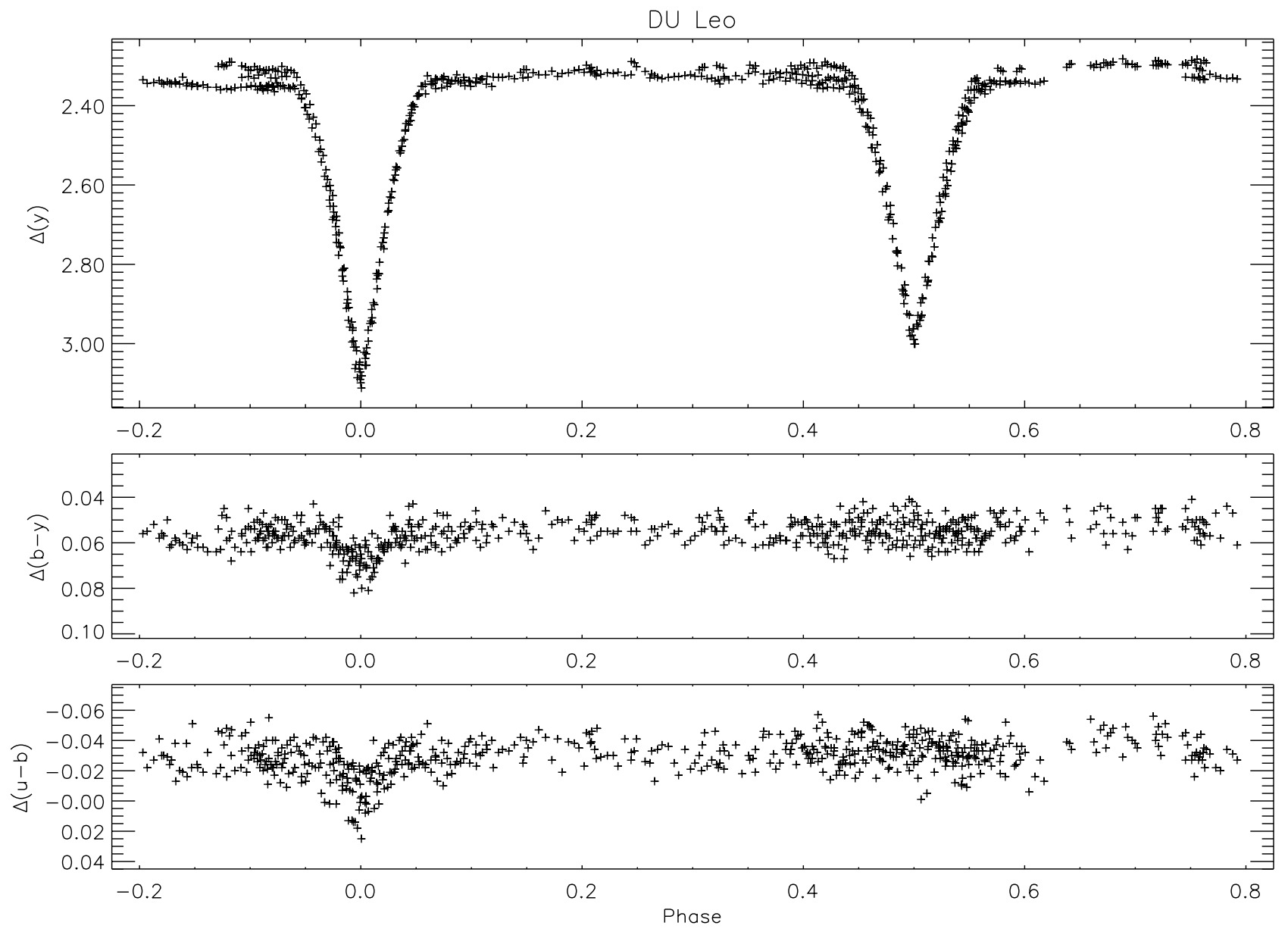

Fig. 8. $y$ light curve and $b-y$ and $u-b$ colour curves (instrumental system) for DU Leo.

Table 9. An extensive list of 42 previous times of minima has been published by Williams (1994), who also derived an improved ephemeris and obtained an orbital period of 1. $37418454 \pm 0.00000006$. Weighted (weight proportional to square of inverse rms) least squares fits to all available times of primary and secondary minima yield periods of $1.3741851 \pm 0.0000003$ and $1.3741846 \pm 0.0000003$, respectively, and nearly identical results are obtained if equal weights are adopted, and if only photoelectric data are used. The results agree well with that by Williams (1994); we have, however, not been able to reproduce the very high accuracy quoted in that paper. We adopt the following ephemeris:

$$
\begin{aligned}
& \text { Min } \mathrm{I}=2450860.66810+1.3741851 \times E . \\
& \pm 6 \quad \pm 3
\end{aligned}
$$

\subsection{Discussion}

The low component masses and the possible high age (Popper 1998a) make DU Leo a very important system for tests of the corresponding stellar models. An additional accurate spectroscopic orbit, based on about 100 radial velocities from the CfA project, has been obtained (Torres 2000, private communication), and five high-resolution spectra ( $R=48000,3800-8600 \AA)$ have been observed with the FEROS spectrograph mounted at the ESO $1.52 \mathrm{~m}$ telescope (Kaufer et al. 1999). These spectra are needed for abundance studies and for determination of the luminosity ratios between the only slightly different components. A detailed study of DU Leo based on all available data will be published separately.

\section{UW LMi}

Olsen (1983) noted HD92823 as a possible variable, and CORAVEL observations (Nordström 1994, private communication) showed it to be double-lined. Based on this information, the uvby indices (Olsen 1983), and a spectral type of G0V, it was included in our search, and during the 1995-96 observing period six low uvby observations were recorded, clearly showing HD92823 to be eclipsing. The Hipparcos mission independently also made this discovery, and a light curve with 100 points is available (ESA 1997, HIP52465). Based on the Hipparcos discovery, the variable name UW LMi was assigned to the object (Kazarovets et al. 1999). A distance of about $130 \mathrm{pc}$ is obtained from the astrometry.

A preliminary spectroscopic orbit (Griffin 1997, private communication; Andersen 1997, private communication) showed that the component masses are $1.14-1.17 M_{\odot}$, and from CORAVEL observations obtained 1997-98, 

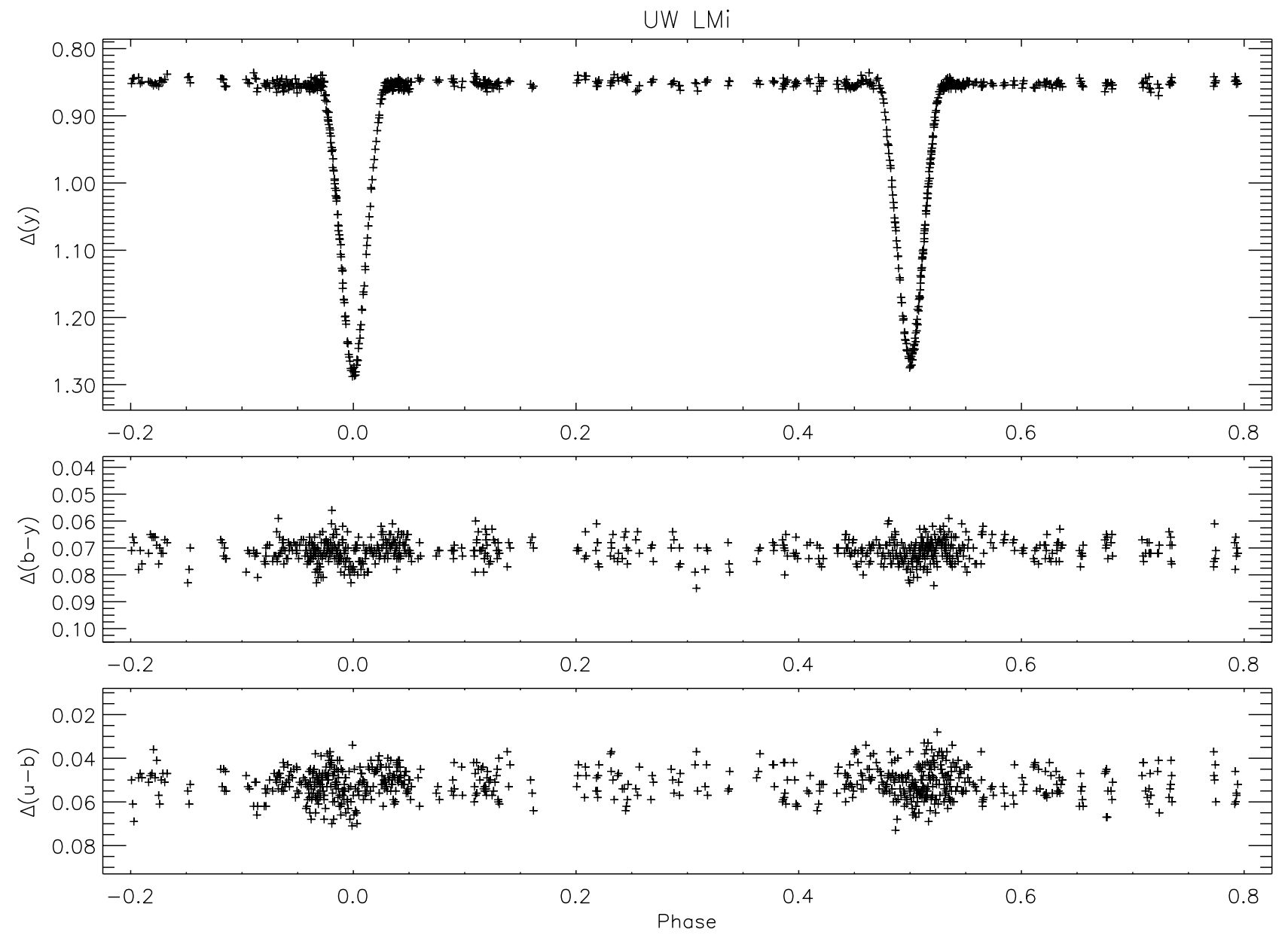

Fig. 9. $y$ light curve and $b-y$ and $u-b$ colour curves (instrumental system) for UW LMi.

Ribas (1999) derived similar results. In total, about 80 radial velocities for each component are available from the CORAVEL project (Udry 1999, private communication).

\section{1. uvby light curves}

Complete uvby light curves (734 points per band) were obtained on 94 nights between JD2450503 and JD2451253. As seen in Fig. 9, UW LMi consists of two well-detached components of similar surface fluxes in a circular orbit. The light curves show no clear indication of surface activity. HD94218, HD94426, and HD91546 were selected as comparison stars and found to be constant within the observational accuracy of a few mmag during our observations; see Tables 1 and 2 for further information.

\subsection{Ephemeris}

From the uvby observations, two times of minimum are available for each eclipse, see Table 10. Orbital periods determined by the method of Lafler \& Kinman (1965) are 3.874307 (all data), 3.874301 (primary eclipse observations), and 3. 874315 (secondary eclipse observations). We adopt the ephemeris:

$$
\text { Min I }=\begin{array}{rrr}
2450854.7742 & +\quad 3.874307 \\
\pm 2 & \pm 7 &
\end{array} \times
$$

Table 10. Times of minima for UW LMi. $O-C$ values are calculated for the ephemeris given in Eq. (8) adopting a circular orbit.

\begin{tabular}{lrcrc}
\hline \hline HJD-2400000 & r.m.s. & Type & $O-C$ & Reference \\
\hline 50854.77420 & 0.00010 & $\mathrm{P}$ & 0.00000 & This paper \\
51226.70700 & 0.00050 & $\mathrm{P}$ & -0.00067 & - \\
50821.84130 & 0.00030 & $\mathrm{~S}$ & -0.00129 & - \\
50922.57470 & 0.00010 & $\mathrm{~S}$ & 0.00013 & - \\
\hline
\end{tabular}

The more massive and slightly hotter component is eclipsed during the marginally deeper primary eclipse at phase 0.0. The ephemeris given in Eq. (8) is far more accurate than that published from the Hipparcos mission (ESA 1997), which erroneously refers to secondary eclipse. It occurs at phase 0.50 , and both the photometric and the spectroscopic analyses show that the orbit is indeed circular.

\subsection{Discussion}

Light curves and radial velocities of very high accuracy have been obtained for the G0V system UW LMi. In addition, four high-resolution spectra $(R=48000$, 3800-8600 A) have been observed with the FEROS 

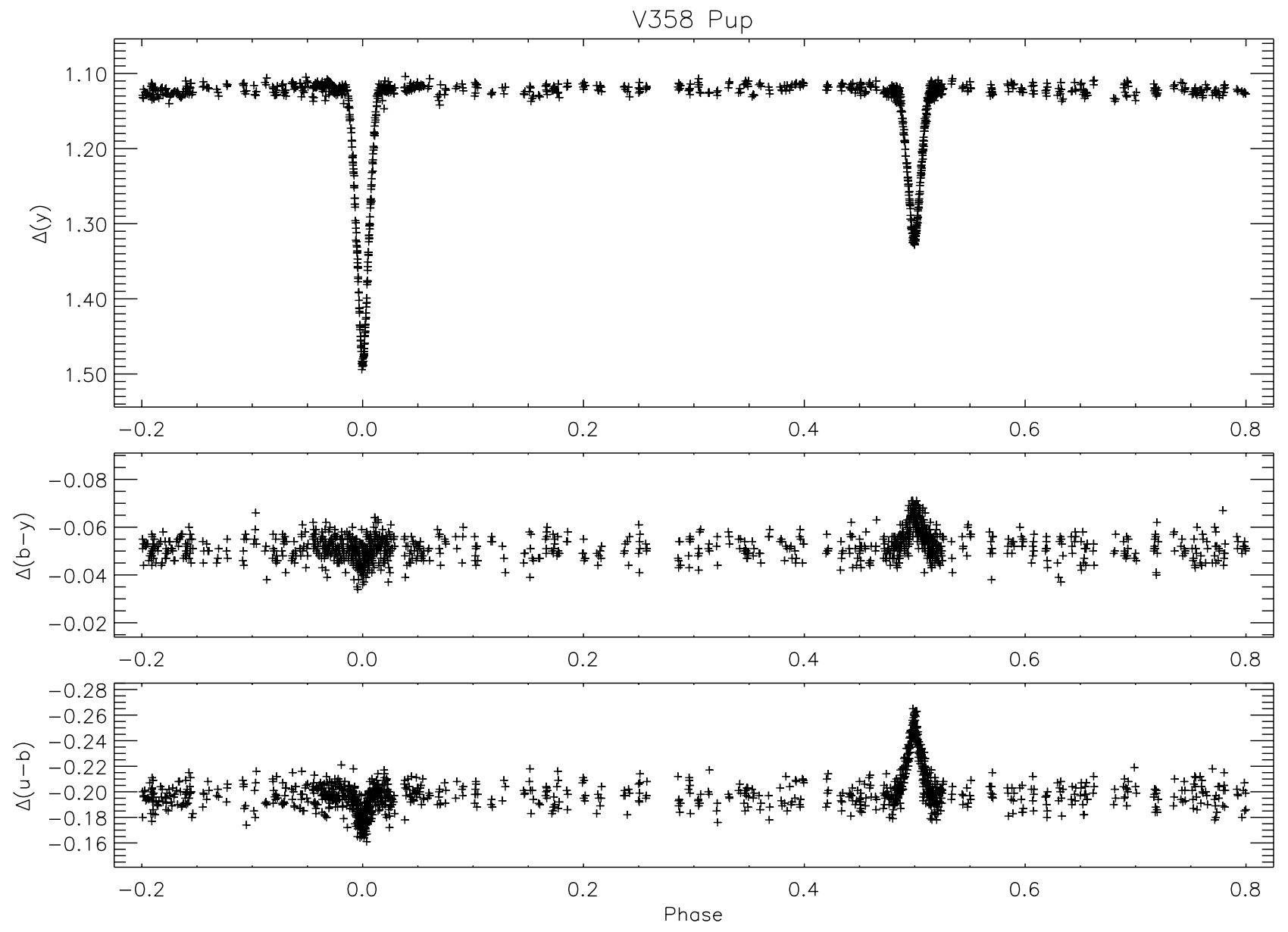

Fig. 10. $y$ light curve and $b-y$ and $u-b$ colour curves (instrumental system) for V358 Pup.

spectrograph mounted at the ESO $1.52 \mathrm{~m}$ telescope (Kaufer et al. 1999). These spectra are needed for abundance studies and for determination of the luminosity ratios between the fairly identical components. A detailed analysis of UW LMi will be published separately (Helt et al. 2001).

\section{V358 Pup}

HD51994 = HIP33487 was discovered by the Hipparcos mission to be an eclipsing binary (ESA 1997, Perryman 1999). 121 Hipparcos photometric observations are available, and about 10 are significantly fainter than the rest. HD51994 subsequently got the variable name V358 Pup (Kazarovets et al. 1999). The ephemeris could, however, not be established from the Hipparcos data. A distance of about $80 \mathrm{pc}$ was obtained. It is a known visual binary, WDS 06577-4118AB $\left(\rho=1^{\prime \prime} .2, \Delta m=0.3\right.$, Worley \& Douglass 1997), for which Hipparcos finds $\rho=1$.'08 and $\Delta m=0.16$. We decided to include V358 Pup in the uvby program in 1997, mainly because of its G5V spectral type.

\section{1. uvby light curves}

Complete uvby light curves (1177 points per band) were obtained on 153 nights between JD2450748 and
JD2451243. HD53522, HD52756, and HD52415 were selected as comparison stars and found to be constant within the observational accuracy of a few mmag during our observations; see Tables 1 and 2 for further information. As seen in Fig. 10, V358 Pup consists of two well-detached and somewhat different components, and the light curves show no indication of pronounced activity. The orbit is very slightly eccentric, see Table 11.

Preliminary analyses of the light curves confirm that the visual companion indeed contributes about $50 \%$ of the total light. Larger fractions of the eclipsing components than indicated by the modest observed depths are therefore obscured during the eclipses.

\subsection{Ephemeris}

From the uvby observations, six times of minimum are available for each eclipse, see Table 11. Weighted (weight proportional to square of inverse rms) least squares fits to the times of primary and secondary minima yield periods of $6.7939254 \pm 0.0000066$ and $6.7939259 \pm 0.0000089$, respectively, and nearly identical results are obtained, if equal weights are used. We adopt the ephemeris:

$$
\text { Min I }=\begin{array}{rrr}
2450818.71458 & +\quad 6 \mathrm{~d} .7939256 & \times E . \\
\pm 20 & \pm 60 &
\end{array}
$$


Table 11. Times of minima for V358 Pup. $O-C$ values (P) and phases (S) are calculated for the ephemeris given in Eq. (9).

\begin{tabular}{lrrrc}
\hline \hline HJD-2400000 & rms & Type & $O-C / \mathrm{Ph}$ & Reference \\
\hline 450784.74575 & 0.00030 & $\mathrm{P}$ & 0.00080 & This paper \\
450818.71420 & 0.00020 & $\mathrm{P}$ & -0.00038 & - \\
451110.85360 & 0.00100 & $\mathrm{P}$ & 0.00022 & - \\
451144.82295 & 0.00020 & $\mathrm{P}$ & -0.00006 & - \\
451151.61695 & 0.00020 & $\mathrm{P}$ & 0.00002 & - \\
451212.76235 & 0.00030 & $\mathrm{P}$ & 0.00009 & - \\
450801.72630 & 0.00020 & $\mathrm{~S}$ & 0.49949 & - \\
450835.69555 & 0.00020 & $\mathrm{~S}$ & 0.49943 & - \\
450869.66590 & 0.00020 & $\mathrm{~S}$ & 0.49954 & - \\
451127.83470 & 0.00030 & $\mathrm{~S}$ & 0.49949 & - \\
451134.62900 & 0.00100 & $\mathrm{~S}$ & 0.49954 & - \\
451236.53670 & 0.00100 & $\mathrm{~S}$ & 0.49937 & - \\
\hline
\end{tabular}

The method of Lafler \& Kinman (1965) confirms the orbital period. Light-time effects due to the visual companion have not been considered, as its (possible) orbit has not yet been established.

\subsection{Discussion}

In addition to the uvby light curves, we have obtained about 35 high-resolution spectra $(R=48000$, 3800$8600 \AA$ ) with the FEROS spectrograph mounted at the ESO $1.52 \mathrm{~m}$ telescope (Kaufer et al. 1999). They will be used for determination of spectroscopic elements and for abundance studies. The spectra, which were observed November 1998 - February 1999, show that the visual companion is a late-type star. Its lines are stronger than those seen from the two eclipsing components. A detailed analysis of V358 Pup will be published separately.

Acknowledgements. It is a great pleasure to thank the many colleagues and students, who have shown interest in our project and have participated in the extensive (semi)automatic observations at the SAT: J. Andersen, J.-P. Beaulieu, R. Clement, F. Derue, J. M. García, R. Haffa, H. Jønch-Sørensen, B. R. Jørgensen, N. T. Kaltcheva, J. Knude, K. K. Knudsen, J. Klougart, S. S. Larsen, A. Lecavelier, C. Ledoux, R. Leguet, C. Loup, M. Maintz, S. Meibom, J. Mouette, D. Mouillet, C. Nitschelm, J. D. Pritchard, J. Suso, A. N. Sørensen, L. L. Vos. Excellent technical support was received from the staff of Copenhagen University and in urgent situations from the $2 \mathrm{p} 2$ team at La Silla. We gratefully acknowledge the CORAVEL and CfA groups for making unpublished radial velocity information and preliminary spectroscopic orbits available. The project "Structure and evolution of stars - new insight from eclipsing binaries and pulsating stars", carried out at Aarhus University and Copenhagen University, is supported by the Danish National Science Research Council. This research has made use of the Simbad database, operated at CDS, Strasbourg, France.

\section{References}

Andersen, J. 1991, A\&A, 3, 91

Cayrel de Strobel, G., Soubiran, C., Friel, E. D., et al. 1997, A\&AS, 124, 299
Clausen, J. V., Helt, B. E., \& Olsen, E. H. 1999a, in Theory and Tests of Convection in Stellar Structure, ed. A. Giménez, E. F. Guinan, \& B. Montesinos, ASP Conf. Ser., 173, 321

Clausen, J. V., Baraffe, I., Claret, A., \& VandenBerg, D. B. 1999b, in Theory and Tests of Convection in Stellar Structure, ed. A. Giménez, E. F. Guinan, \& B. Montesinos, ASP Conf. Ser., 173, 265

Clausen, J. V., Ribas, I., Helt, B. E., Hinse, T. C., \& Wolf, J. A. 2001, A\&A, in prep.

Cutispoto, G., Kürster, M., Messina, S., Rodonò, M., \& Tagliaferri, G. 1997, A\&A, 320, 586

ESA 1997, The Hipparcos and Tycho Catalogues, ESA SP-1200

Germain, M. E., Douglass, G. G., \& Worley, C. E. 1999, AJ, 117,1905

Helt, B. E., Ribas, I., Griffin, R. F., \& Clausen, J. V. 2001, A\&A, in prep.

Kaiser, D. H. 1990, Inf. Bull. Variable Stars, 3480

Kaufer, A., Stahl, O., Tubbesing, S., et al. 1999, The ESO Messenger, 95, 8

Kazarovets, E. V., Samus, N. N., \& Goranskij, V. P. 1993, Inf. Bull. Variable Stars, 3840

Kazarovets, E. V., Samus, N. N., Durlevich, O. V., et al. 1999, Inf. Bull. Variable Stars, 4659

Kwee, K. K., \& van Woerden, H. 1956, BAN, 12, 327

Lafler, J., \& Kinman, T. D. 1965, ApJS, 11, 216

Lockwood, G. W., Skiff, B. A., \& Radick, R. R. 1997, ApJ, 485,789

Lu, W.-X. 1986, PASP, 98, 468

Marschall, L. A., Gauthier, C. P., Nations, H. L., Taylor, B. W., \& Huard, T. L. 1991, Inf. Bull. Variable Stars, 3633

Nations, H. L., Huard, T. L., Marschall, L. A., et al. 1991, BAAS, 23, 1414

Olsen, E. H. 1983, A\&AS, 54, 55

Olsen, E. H. 1988, A\&A, 189, 173

Olsen, E. H. 1993, A\&AS, 102, 89

Olsen, E. H. 1994a, A\&AS, 104, 429

Olsen, E. H. 1994b, A\&AS, 106, 257

Olsen, E. H., Clausen, J. V., \& Helt, B. E. 2001a, A\&A, in prep.

Olsen, E. H., Clausen, J. V., \& Helt, B. E. 2001b, A\&A, in prep.

Perryman, M. 1999, Sky Telesc. 97, 6, 40

Popper, D. M. 1993, ApJ, 404, L67

Popper, D. M. 1994, AJ, 108, 1091

Popper, D. M. 1995, Inf. Bull. Variable Stars, 4185

Popper, D. M. 1996, ApJS, 106, 133

Popper, D. M. 1997a, AJ, 113, 1457

Popper, D. M. 1997b, AJ, 114, 1195

Popper, D. M. 1998a, AJ, 115, 338

Popper, D. M. 1998b, PASP, 110, 919

Popper, D. M. 2000, AJ, 119, 2391

Pye, J. P., McGale, P. A., Allan, D. J., et al. 1995, MNRAS, 274,1165

Ribas, I. 1999, Ph.D. Thesis, Universitat de Barcelona

Robb, R. M., Dean, F. W., \& Scarfe, C. D. 1990, Inf. Bull. Variable Stars, 3536

Schuster, W. J., \& Nissen, P. E. 1988, A\&AS, 73, 225

Strohmeier, W., Knigge, R., \& Ott, H. 1964, Inf. Bull. Variable Stars, 74

Williams, D. B. 1994, Inf. Bull. Variable Stars, 3999

Williams, D. B., Baldwin, M. E., \& Kaiser, D. H. 1990, Inf. Bull. Variable Stars, 3514

Worley, C. E., \& Douglass, G. G. 1997, A\&AS, 125, 523 